\title{
Real-Time Acousto-Optic Visualization of Ultrasonic Pulses in $\mathrm{TeO}_{2}$
}

\author{
O. Polishko*, A. Yurchenko, Y. Pilgun and E. Smirnov \\ Department of Quantum Radiophysics, Taras Shevchenko National University of Kyiv, \\ 64/13 Volodymyrska St., 01601 Kyiv, Ukraine
}

\begin{abstract}
The ultrasonic beam features in ultrasonic and acousto-optic devices have a great impact on their characteristics and need to be studied. In the current work, a conventional optical visualization technique was extended to include spatial filtration of the light diffraction pattern and stroboscopic illumination of a unit under test. That enabled very weak light responses from ultrasonic waves to be observed. This technique was applied to studying propagation and reflection of ultrasonic pulses in the $\mathrm{TeO}_{2}$ crystal, a body of the total internal reflection ultrasonic sensor. Analysis of visualization data has revealed an unexpected conversion of the shear horizontal wave radiated by the transducer into a longitudinal one on its normal reflection from the (001) face of the crystal, which is inexplicable as far as the plane wave theory is concerned. This finding requires further research to be confirmed and explained.
\end{abstract}

DOI: 10.12693 /APhysPolA.127.132

PACS: $43.20 . \mathrm{Gp}, 43.35 . \mathrm{Sx}$

\section{Introduction}

Recent researches [1, 2] show that ultrasonic beam features have a great impact on a design of ultrasonic devices including acousto-optic (AO) ones [3]. Real-time visualization of ultrasonic pulses in an anisotropic medium enables to clarify physical mechanisms governing behaviour of ultrasonic beams.

The Schlieren imaging technique is a well-known method for visualization of acoustic fields [4-7]. It is based on the use of light diffraction caused by acoustic waves. The intensity of the diffracted light depends on the value of an effective photoelastic coefficient that in some cases can be equal to zero, which is considered forbidden for acousto-optic interaction. Nevertheless, a weak AO interaction near a "forbidden direction" was observed in experiments [8].

The purpose of the current research is to study propagation and reflection of ultrasonic pulses in the total internal reflection ultrasonic sensor (TIRUS) [9] made of a $\mathrm{TeO}_{2}$ crystal.

Unlike the case considered in [9] where the slow shear wave (SSW) was emitted into the [110] direction, in the current research it was emitted into the [001] direction (Fig. 1). Visualization of acoustic fields in the sensor was carried out in the (1110) plane, in which case the crystal should be illuminated from the forbidden direction.

\section{Experimental technique and the sample examined}

In the experiments, the shear acoustic wave polarized along the [110] direction was emitted from the top face of

\footnotetext{
* corresponding author; e-mail: alexanderrff@gmail.com
}

the crystal by a $163^{\circ} Y$-cut $\mathrm{LiNbO}_{3}$ transducer (Fig. 1). An examined experimental sample of the TIRUS was a rectangular bar of $23 \times 12 \times 10 \mathrm{~mm}^{3}$ in size with one (110) face tilted at the angle of $16.25^{\circ}$ with respect to the [110] direction. The faces (001), (11̄0), and (110) were oriented with accuracy not worse than \pm 2 arcmin with respect to corresponding crystallographic directions. The faces (110) had antireflection coatings for the $532 \mathrm{~nm}$ wavelength.

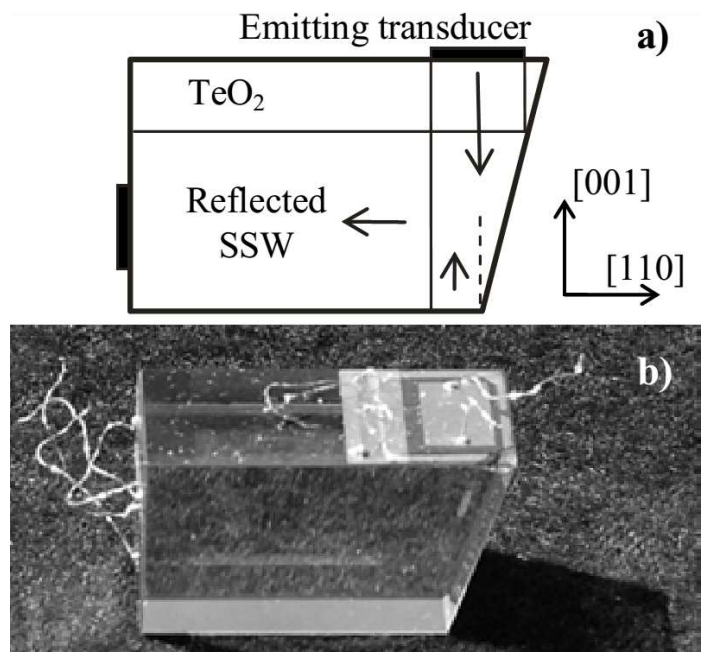

Fig. 1. A schematic (a) and an appearance (b) of the experimental sample.

As seen from Fig. 1a, a projection of the reflecting oblique face of the crystal on its top face is smaller than the size of the top transducer. As a result, only a part of the pulse emitted is reflected from this face whereas its another part is reflected from the bottom face. This leads to interesting effects to be discussed further. 


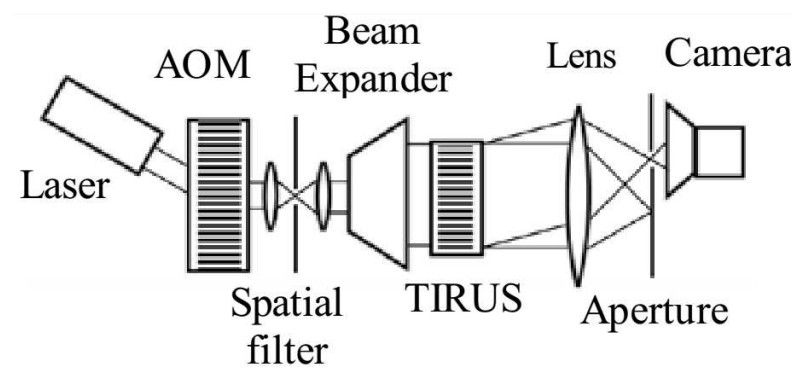

Fig. 2. A sketch of the optical setup.

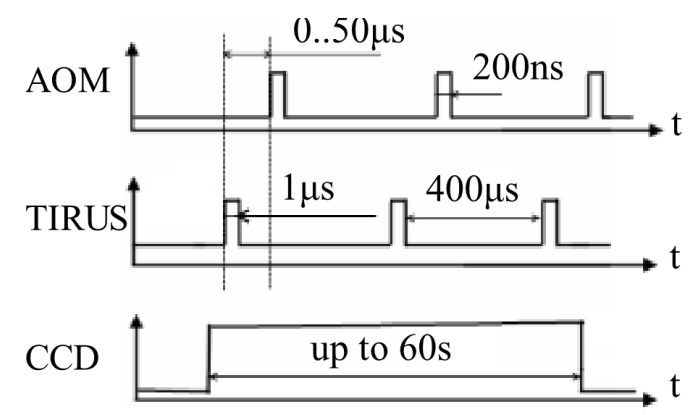

Fig. 3. Time diagram of controlling pulses.

The experimental setup is shown in Fig. 2. A laser with a wavelength of $532 \mathrm{~nm}$ is used as a light source. The laser beam is modulated with an acousto-optic modulator (AOM) by $200 \mathrm{~ns}$ pulses. The modulated laser beam passes through a spatial filter and a beam expander illuminating the sensor body by short well-collimated light pulses. The top transducer emits a sequence of the $1 \mu \mathrm{s}$ SSW pulses at the carrier of $27 \mathrm{MHz}$. The sequence period is $400 \mu \mathrm{s}$, therefore all previously excited pulses in the crystal are completely decayed. A predetermined time delay between the light and ultrasonic pulses allows to illuminate acoustic field at a certain moment after emitting the ultrasonic pulse (Fig. 3). The laser beam is diffracted by ultrasonic waves and a diffraction order of interest is selected by an aperture in the focal plane of the collecting lens. The light passing the aperture is recorded by a sensitive CCD camera. Its exposure time can be chosen long enough, up to $60 \mathrm{~s}$, during which it collects over 100,000 light pulses. Taking pictures at sequential moments of time, one can trace propagation of pulses in the crystal.

If the selected acoustic wave has a high velocity, the diffracted light beam is close to the incident one, so the amount of noise passing through the aperture is significant. Thus, the aperture size determines not only an angular spectrum of acoustic waves to be mapped, but also the signal to noise ratio. In the experiments, it was improved by means of subtracting preliminary recorded images of a background from the recorded images of ultrasonic pulses. Saturation of some areas in the background image led to corresponding black areas in the resultant difference image. As a result, a chosen exposure time had to be a compromise between an acceptable number of the underexposed details to be obtained and an acceptable size of these areas. Therefore, additional post-processing, including colorizing resultant images, was necessary.

\section{Results}

In this work we investigated reflection of the SSW pulses propagating along the [001] direction in the sensor body. The shear wave transducer generated not a pure but a quasi-shear wave, therefore in the crystal not one but two acoustic modes were excited, a spurious longitudinal and a shear wave. As a result, two ultrasonic waves with different polarizations were emitted simultaneously (Fig. 4a). They were observed in two different series of experiments where either a longitudinal or a shear mode was selected. The recorded images are presented side-byside to provide full information on propagating pulses. The shear wave generated by the top transducer was polarized along the [110] direction, that is, its polarization was perpendicular to the plane of the picture; so it was of a shear horizontal $(\mathrm{SH})$ type of the wave with respect to the top, bottom, and oblique faces of the crystal. Thus, its displacement vector lay in the plane of these faces when the wave struck them on reflection.

Intensity of the longitudinal wave is much less than that of the shear wave, but intensity of light diffracted by it is significantly greater than that for the shear wave; it is so because for the longitudinal wave the acoustooptic figure of merit $M_{2}=34.5 \times 10^{-15} \mathrm{~s}^{3} / \mathrm{kg}$ [10] against the theoretically zero value of that for the observed shear wave. AO interaction for the longitudinal wave was observed when the incident light beam propagated precisely along the [110] direction because the Bragg angle was less than the divergence angle of the ultrasonic beam. At the same time, the crystal had to be rotated around the [110] direction by $\approx 2^{\circ}$ to visualize the shear wave.

As seen in Fig. 4, the right parts of radiated ultrasonic pulses fall onto the oblique face of the crystal and are reflected into the [110] direction whereas their left parts fall onto the bottom face of the crystal. The velocities of the emitted shear and longitudinal waves are 2103 and $4203 \mathrm{~m} / \mathrm{s}$, respectively, so the longitudinal wave pulse is roughly twice faster than the shear one, therefore it returns back to the transducer just at the moment when the shear wave pulse reaches the bottom (Fig. 4b). The displacement vector of the incident shear pulse lies in the bottom face of the crystal. Therefore, only a reflected shear pulse has to be a result of the incident wave reflection from the bottom. However, as seen in Fig. 4, right column, a new longitudinal pulse is radiated from the bottom simultaneously with reflection of the shear pulse (Fig. 4, left column). Because the top and bottom faces of the crystal are oriented with respect to the [001] direction with accuracy not worse than \pm 2 arcmin, any normal components of the incident shear wave displacement can be neglected. So the only visible reason for the emerging longitudinal pulse is conversion of the incident 

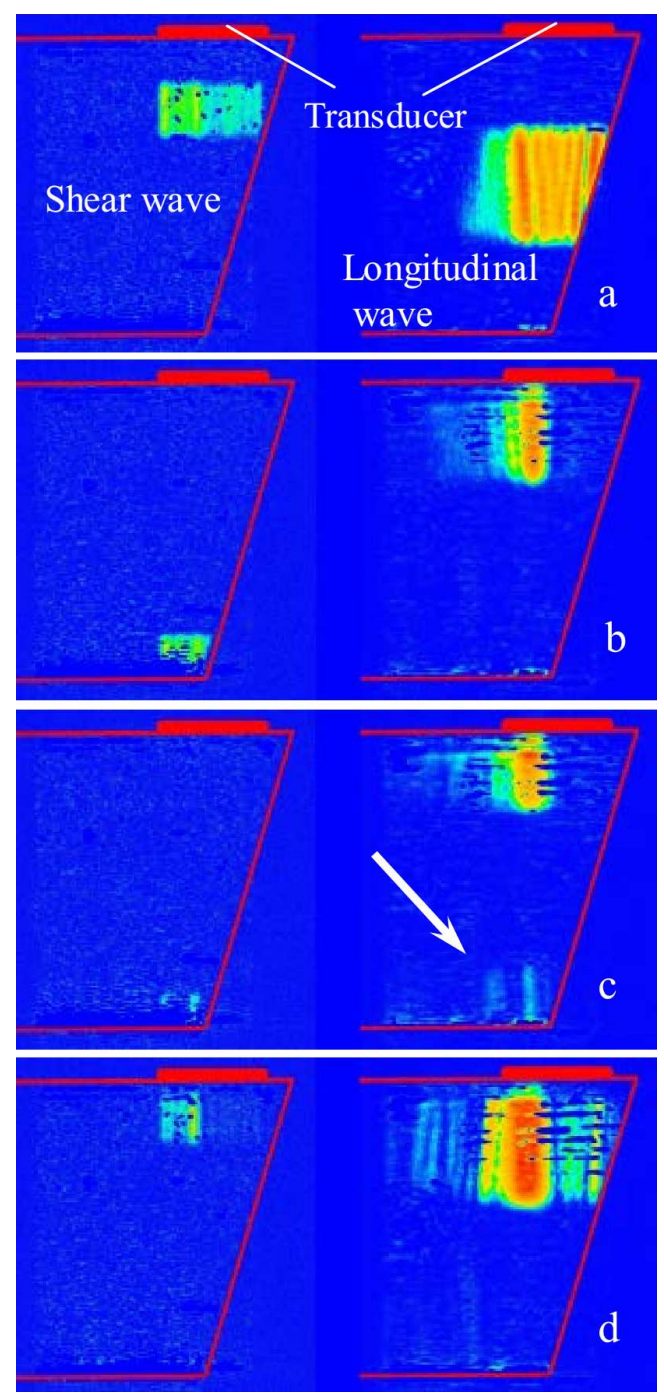

Fig. 4. Shear (left) and longitudinal (right) pulses propagating along the [001] direction in the TIRUS. Pictures are taken at $1.9 \mu \mathrm{s}(\mathrm{a}), 5.8 \mu \mathrm{s}(\mathrm{b}), 6.4 \mu \mathrm{s}$ (c), and $12.6 \mu \mathrm{s}(\mathrm{d})$ time points.

shear wave into a longitudinal one, because there are no other "sources" except it at the bottom at this moment (Fig. 4b). The newly born longitudinal pulse (indicated with an arrow in Fig. 4c) has the same duration as a pulse radiated from the transducer, but it propagates in the opposite direction. One can see, however, that its group velocity deflects from the [001] direction by $\approx 5^{\circ}$ in the (110) plane. Therefore, after a few reflections, the new pulse leaves the observed area.

A possible reason for this observed theoretically impossible for plane waves conversion might be conversion of some plane-wave spectral components of the incident ultrasonic beam that propagates close to the [001] direction. The displacement vectors of these wave components might lie out of the bottom and consequently can have [001] components generating a longitudinal wave.

Further the propagation-reflection process develops as follows. The SSW pulse returns to the top transducer after $12 \mu \mathrm{s}$ from its starting time from the transducer and re-excites it. The transducer re-emits a new pair of shear and longitudinal pulses again, which overlap with the returning ones (Fig. 4d). All the process described repeats cyclically decaying step-by-step because the energy of the shear pulse is partly being lost on every reflection from the oblique surface into the [110] direction.

\section{Conclusions}

A time gated procedure combined with the spatial filtration of the entire light diffraction pattern developed for visualization of acoustic waves in crystals allows to observe and study propagation and reflection of ultrasonic pulses with a high degree of accuracy and sensitivity. As a result, propagation of ultrasonic pulses could be observed from the "forbidden direction" and the spatial structure of the slow shear wave could be visualized. Analysis of the visualization data has revealed an unexpected conversion of the radiated $\mathrm{SH}$-wave into a longitudinal one on its reflection from the (001) face of the crystal, which is inexplicable as far as the plane wave theory is concerned. This finding requires further research including both an experimental confirmation of the observed conversion and a theoretical analysis of spatial properties of the incident ultrasonic beam including calculation of its angular spectrum.

\section{Acknowledgments}

This material is based upon work supported by the European Office of Aerospace Research and Development, AFOSR, AFRL as the STCU Partner Project P475.

\section{References}

[1] L.N. Magdich, Yu.V. Pisarevskii, N.N. Semenovskii, O.Yu. Sil'vestrova, J. Commun. Technol. Electron. 53, 1442 (2008).

[2] E.A. D'yakonov, V.B. Voloshinov, N.V. Polikarpova, Acoust. Phys. 58, 107 (2012).

[3] N. Naumenko, S. Chizhikov, V. Molchanov, K. Yushkov, Proc. IEEE IUS 2013, Ed. J. Saniee, IEEE, Prague 2013, p. 500.

[4] M.A. Breazeale, Proc. SPIE 3581, 41 (1998).

[5] T. Neumann, H. Ermert, Ultrasonics 44, e1561 (2006).

[6] M.A. Breazeale, J. Opt. A Pure Appl. Opt. 3, S1 (2001).

[7] D. Mehrl, A. Korpel, W. Bridge, Appl. Opt. 29, 4766 (1990).

[8] L. Voitenko, E. Smirnov, A. Yurchenko, Bull. Taras Shevchenko Natl. Univ. Kyiv Phys. Math. 11, 13 (2008).

[9] A. Yurchenko, V. Danilov, Yu. Pilgun, E. Smirnov, Proc. IEEE IUS 2013, Ed. J. Saniee, IEEE, Prague 2013, p. 190.

[10] N. Uchida, Y. Ohmachi, J. Appl. Phys. 40, 4692 (1969). 